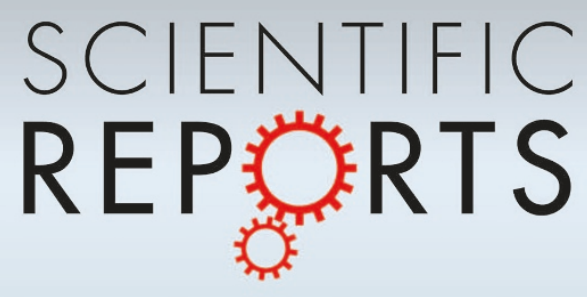

\title{
$-$ \\ NIRS external dose estimation system for Fukushima residents after the Fukushima \\ SUBJECT AREAS: \\ SOFTWARE Dai-ichi NPP accident
}

PUBLIC HEALTH

RISK FACTORS

ENVIRONMENTAL SCIENCES

Received

21 February 2013

Accepted

2 April 2013

Published

17 April 2013

Correspondence and requests for materials should be addressed to

K.A. lakahane@nirs.

go.jp)

Keiichi Akahane, Shunsuke Yonai, Shigekazu Fukuda, Nobuyuki Miyahara, Hiroshi Yasuda, Kazuki Iwaoka, Masaki Matsumoto, Akifumi Fukumura \& Makoto Akashi

National Institute of Radiological Sciences, 4-9-1, Anagawa, Inage-ku, Chiba-shi 263-8555 Japan.

The great east Japan earthquake and subsequent tsunamis caused Fukushima Dai-ichi Nuclear Power Plant (NPP) accident. National Institute of Radiological Sciences (NIRS) developed the external dose estimation system for Fukushima residents. The system is being used in the Fukushima health management survey. The doses can be obtained by superimposing the behavior data of the residents on the dose rate maps. For grasping the doses, 18 evacuation patterns of the residents were assumed by considering the actual evacuation information before using the survey data. The doses of the residents from the deliberate evacuation area were relatively higher than those from the area within $20 \mathrm{~km}$ radius. The estimated doses varied from around 1 to $6 \mathrm{mSv}$ for the residents evacuated from the representative places in the deliberate evacuation area. The maximum dose in 18 evacuation patterns was estimated to be $19 \mathrm{mSv}$.

(T)

he great east Japan earthquake occurred at 14:46, Japan time on 11 March 2011 in the Tohoku District, northeastern part of Japan. After the quake, big tsunamis rushed to the coast of the Tohoku district including Fukushima prefecture. The tsunamis caused electric failure of Fukushima Dai-ichi Nuclear Power Plant (NPP). The cooling systems of nuclear reactors stopped, and the hydrogen explosions occurred. Subsequently, radioactive fission products leaked out to the environment ${ }^{1,2}$.

The interest in radiation doses due to the accident has been increasing among the Fukushima residents. The importance to estimate exposure doses of the residents has been recognized among the involved organizations. National Institute of Radiological Sciences (NIRS) started to develop the external dose estimation system for Fukushima residents at the end of March, 2011. On the other hand, Fukushima prefectural government and Fukushima Medical University decided to perform the health management survey for all Fukushima residents (about two million people) at the end of May, 2011 ${ }^{3}$. External dose was considered to be one of the necessary data for the health management, and then the NIRS external dose estimation system was adopted in the survey.

This paper describes the NIRS external dose estimation system and the calculation results for 18 evacuation patterns assumed by NIRS.

\section{Results}

We developed the NIRS external dose estimation system for the Fukushima residents to estimate the external effective doses for the first four months after the Fukushima Dai-ichi nuclear power plant accident. This system has been adopted in the Fukushima Health Management Survey, and the estimated result is sequentially provided to the individual Fukushima residents.

The estimated external doses of the adult residents in 18 evacuation patterns were shown in Table 1 . The results show that the doses of the residents evacuated from the representative places in the deliberate evacuation area (typically 1-6 mSv) were relatively higher than those from the area within $20 \mathrm{~km}$ radius (around $1 \mathrm{mSv}$ ). The maximum dose in 18 evacuation patterns was estimated to $19 \mathrm{mSv}$.

\section{Discussion}

The NIRS external dose estimation system for the Fukushima residents has been developed based on the simulation with the System for Prediction of Environmental Emergency Dose Information (SPEEDI) ${ }^{4}$ and monitoring data reported officially by the government. The uncertainties in this dose estimation depend mainly on not only those of the dose rate map but also the residents' memory on the behavior. 
Table 1 | Estimated external effective doses (mSv) for adult of 18 patterns

Estimated external effective doses for adult (mSv)

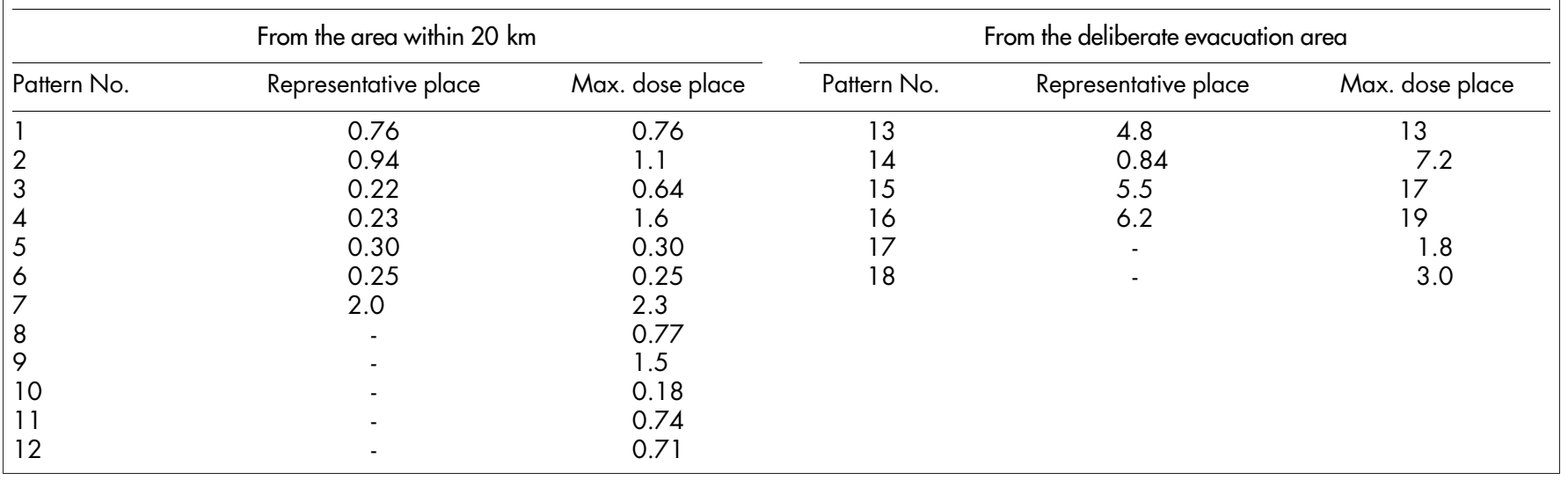

With regard to the dose rate map, since maps for the first 3 days are based on the SPEEDI simulation instead of the monitoring data, the uncertainty is expected to be higher than those for other days. We have no alternatives for first 3 days at present because of the scarce monitoring data. The calculated results of SPEEDI greatly depend on the source term emitted from the nuclear reactors, which is expressed as a function of time. In the current situation, we have used the available and reliable data of the source term reported by the government. However, when the accuracy improvement of the simulation is done with further analysis on the source term, recalculations with the dose rate maps base on the newly-simulated results would be necessary. On the other hand, the contributions of exposures in early stage for total estimated doses are not so much, because most residents near the NPP have evacuated early after the accident and the increases of the dose by radionuclides in the plume were limited for a few hours as shown in Fig. 1. Actually the dose by radionuclides deposited on the ground etc., which is relatively plateau for the four months from the accident, is more important. For example, even at the Minami-soma city office where the increase of dose was observed earlier than other areas as shown in Fig. 1, the contribution of the first 3 days to the total dose of four months was estimated to be about 5\% by the NIRS system and about $10 \%$ when using the monitoring data. This fact indicated that the refinement of the simulation for the first 3 days would not so affect to the total dose.

In the NIRS system, the conversion coefficients from ambient dose equivalents to effective doses for isotropic irradiation were adopted. ICRP does not provide the conversion coefficients for the special condition of the irradiation. Saito et al. $^{8}$ calculated the conversion coefficients of effective dose from environmental gamma ray sources: cloud and ground shines. The coefficients of isotropic irradiation (ISO), lateral irradiation from the right side to the left side of the body (RLAT) and lateral irradiation from the left side to the right side of the body (LLAT) are close to that assumed gamma ray source on the ground according to ICRP $74^{\circ}$ and Saito el al., and the coefficient of ISO is the most conservative. Table 2 shows the conversion coefficients from ambient dose equivalent to effective dose for adult for

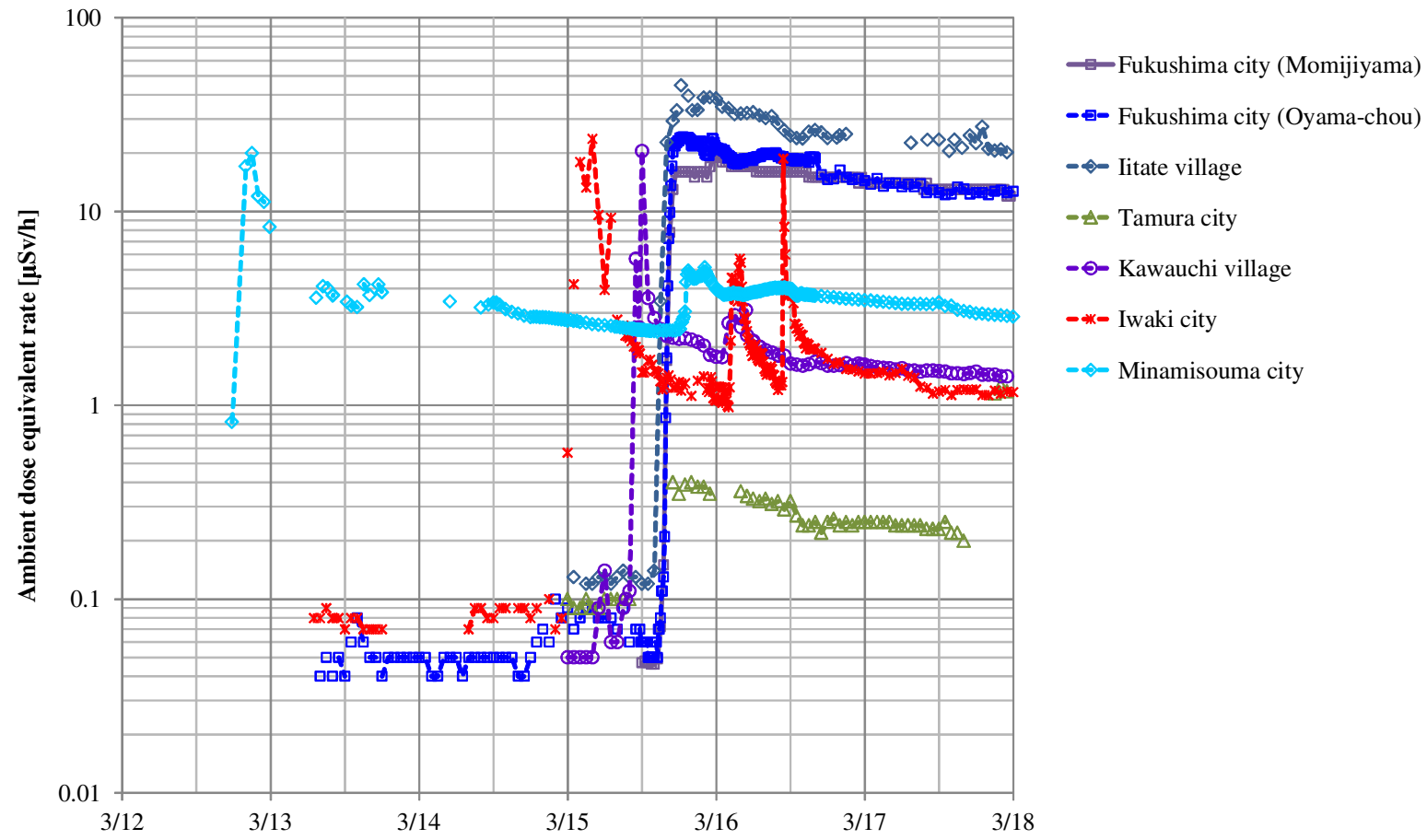

Figure $1 \mid$ Monitoring data reported by MEXT and Fukushima prefectural government ${ }^{5,6,7}$. 
Table 2 | Conversion coefficients from ambient dose equivalent to effective dose for adult for various radionuclides

\begin{tabular}{|c|c|c|c|c|c|c|c|}
\hline Nuclide & $X e-133$ & Te- $129 \mathrm{~m}$ & Te-132 & $\mid-131$ & $\mid-132$ & $\mathrm{Cs}_{\mathrm{S}-134}$ & Cs-137 \\
\hline $\begin{array}{l}\text { Saito et al. }{ }^{8} \\
\text { (Ground) }\end{array}$ & 0.41 & 0.61 & 0.51 & 0.57 & 0.62 & 0.61 & 0.61 \\
\hline $\begin{array}{l}\text { Saito et al. } \\
\text { (Air) }\end{array}$ & 0.34 & 0.57 & 0.46 & 0.52 & 0.58 & 0.57 & 0.57 \\
\hline Present & 0.44 & 0.58 & 0.48 & 0.53 & 0.59 & 0.58 & 0.57 \\
\hline
\end{tabular}

various radionuclides considered in this study calculated from ICRP 74 (adopted in this study) and Saito el al. This result indicated that conversion coefficient of 0.6 used in the NIRS system is reasonable.

\section{Methods}

Flow of the dose calculation. In the NIRS system, the external effective dose between March 12 and July 11, 2011 is estimated by superimposing the individual behavior data of each day on the daily dose rate map of such day. The data flow in the external dose estimation system is shown in Fig. 2. The flow is composed of three parts: 1) behavior data of residents, 2) construction of daily dose rate maps, and 3) calculation of effective dose. Each part is described below. This system utilizes two kinds of the fundamental data: daily dose rate map and behavior data of the resident. The background dose due to natural radiation sources was not included in the dose rate maps, because of the radiation protection purposes. The body size as a function of age is also considered because the external effective dose depends on body size even in the same radiation field. The dose rate maps have the data of effective dose for adults, so body size correction is considered for children less than or equal to 15 years old by using age coefficients.

Data of behaviors of the residents. Fukushima prefectural government has been conducting the health management survey for the Fukushima residents to support their health conditions affected by the accidents in cooperation with Fukushima Medical University ${ }^{3}$. The survey consists of two kinds of investigations, which are called the basic survey and the detailed survey, respectively. In the basic survey, questionnaires regarding behavior of a resident for four months after the accident were sent by postal mail to the all residents in Fukushima prefecture, about 2 million in the population. Based on such data, the behavior data of the resident for each day can be obtained as follows;

- $\quad$ Place to stay, $i$,

- Time of stay in the building at $i, t_{i n, i}$,

- Time of stay outside the building at $i, t_{\text {out }, i \text {, }}$

- Type of the building at $i, k_{i}$,

- Time of the move from $i$ to $i+1, t_{m o v e, i \rightarrow i+1}$, (if any)

Construction of daily dose rate maps. The dose rate maps consisting of a lot of divisions were made based on the simulated and measured data in a time series between 12 March and 11 July in 2011. The size of each division in the map is corresponding to approximately $2 \mathrm{~km} \times 2 \mathrm{~km}$, which is based on the size divided the second mesh (latitude distance of $7.5 \mathrm{~min}$. by longitude distance of $5 \mathrm{~min}$ ) defined by Geospatial Information Authority of Japan.

The daily dose rate maps were constructed based on two kinds of data. One is the hourly effective dose rate maps simulated by SPEEDI with the source term calculated by the MELCOR code ${ }^{10}$ by the Nuclear and Industrial Safety Agency (NISA), which was used between March 12 and 14, 2011 ${ }^{11}$. These data are an alternative to the monitoring data, because the number of measurement point was not sufficient to construct the dose rate maps in the period. The hourly effective dose rate maps outputted from the SPEEDI simulations, were averaged over a day, because the dose rate maps used in our system were daily maps. Also, its area was divided to those of $1 \mathrm{~km} \times 1 \mathrm{~km}$. Therefore, the dose rate maps generated by SPEEDI were reconstructed by dividing in proportion to the area size of our system (approximately $2 \mathrm{~km}$ $\times 2 \mathrm{~km}$ ) with commercially-available mapping software. As a result, the effective dose (for adult) rate maps with $d(h, m)$, where $h$ and $m$ are the date and the division number, respectively, were obtained between 12 and 14 March, 2011. Unfortunately, their area was limited in $98 \mathrm{~km} \times 98 \mathrm{~km}$, which is painted in green in Fig. 3, depending on the SPEEDI ability. At present, the doses are not added outside of the area.

Another data is monitoring data released by the Ministry of Education, Culture, Sports, Science and Technology (MEXT), which was used between March 15 and July 11,2011 . The monitoring data scattered in the map were converted to the

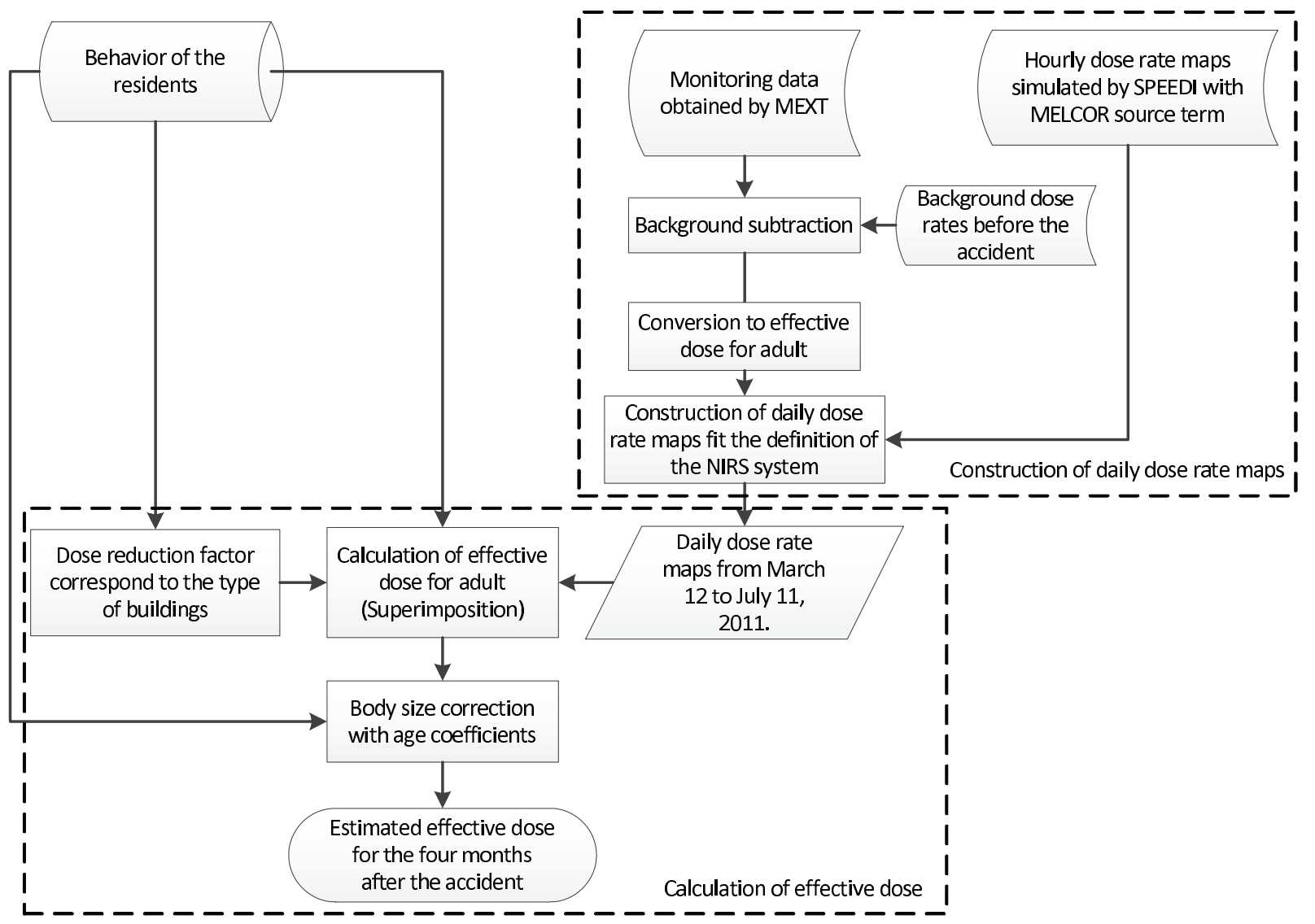

Figure $2 \mid$ Data flow in the NIRS external dose estimation system. 


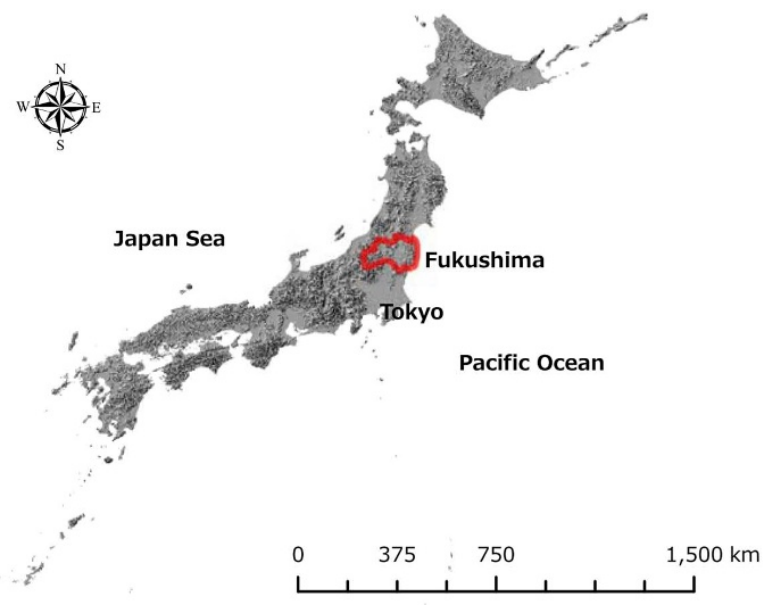

March 12-14, 2011

On/After March 15, 2011
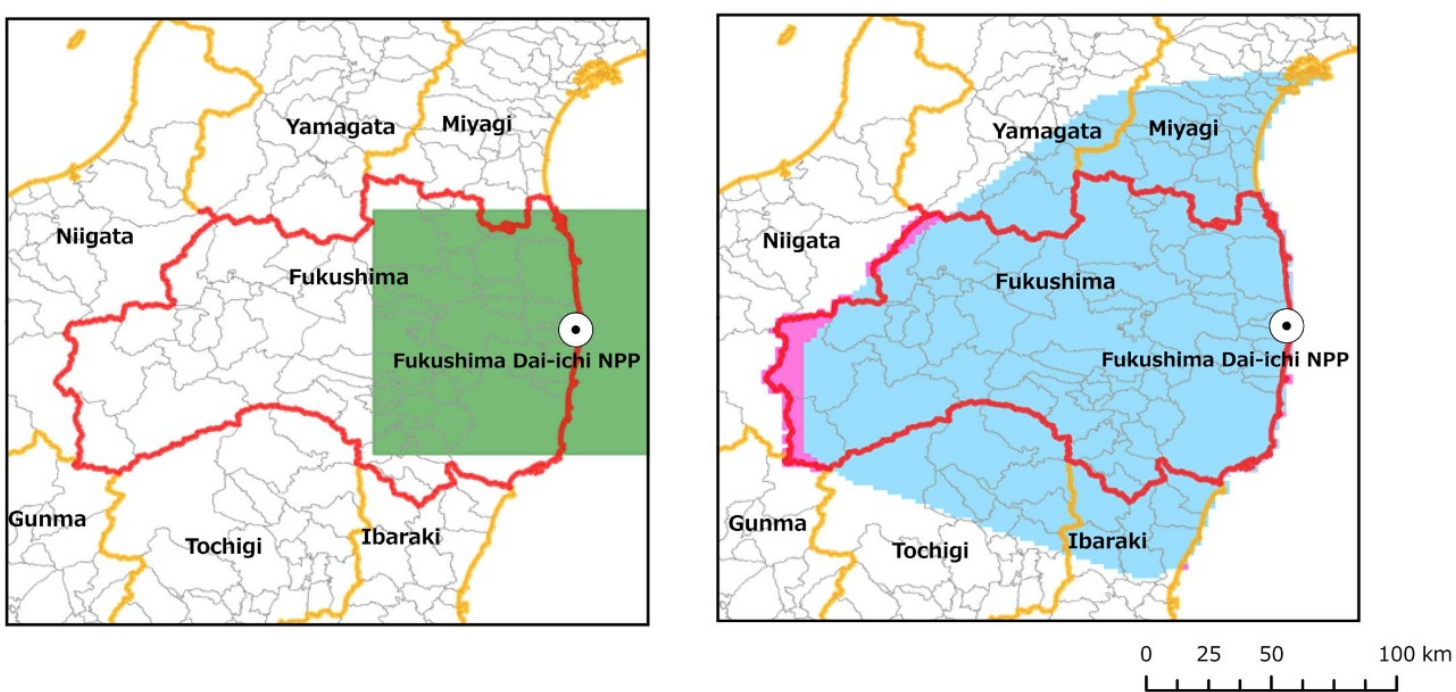

Figure 3 Areas of dose rate maps used in the NIRS external dose estimation system. Basic maps were created by using ArcGIS of ESRI.

spatially-continuous data by using Natural Neighbor method and then the daily dose rate in each division of approximately $2 \mathrm{~km} \times 2 \mathrm{~km}$ was obtained by averaging the values in such division. Since the monitoring data maps by MEXT could not cover the area of a part of seaside and the boundary between Fukushima and Niigata prefectures (pink-colored areas in Fig. 3), the values of their neighbor on the right or left were used alternatively.

Monitoring data on 15 March, 2011 were not sufficient to construct the dose rate map. On the other hand, this SPEEDI results were significantly different from the monitoring data. This was expected to derive from the highest time variation of the dose rate in Fig. 1. Therefore, the dose rate map on 16 March was alternatively used for that on 15 March. Table 3 shows the comparison of effective doses on 15 and 16 March, 2011 based on the monitoring data at the points shown in Fig. 1. The differences were between -11 and $+182 \mu \mathrm{Sv}$ (effective dose), so the alternative does not lead to a significant underestimation. In fact, it leads to an overestimation at most points.

In the NIRS system, the background dose from natural radionuclides, etc. is not included in the estimated dose. Therefore, the background dose was estimated from the reported data before the accident. Fukushima prefectural government has

Table 3 | Comparison of effective dose ( $\mu \mathrm{Sv})$ on 15 and 16 March, 2011 based on the monitoring data

Effective dose $(\mu \mathrm{Sv})$

Measurement point

16 March, 2011 (B)

Fukushima city (Momijiyama)

Fukusima city (Oyama-chou)

Difference (B-A)

litate village

Tamura city

70



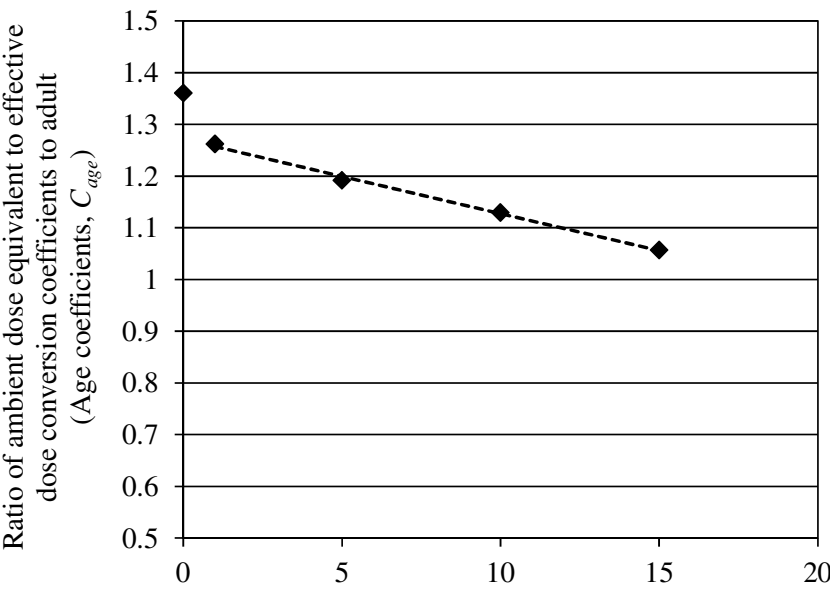

Age, $y$ [year]

Figure $4 \mid$ Ratios of conversion coefficients for each age group to adult.

reported the averaged air kerma rates per month at 23 points in the prefecture ( 1 in Kouno town, 4 in Naraha town, 5 in Tomioka town, 5 in Okuma town, 4 in Futaba town and 4 in Namie town) as 33-54 $\mu \mathrm{Gy} / \mathrm{h}^{12}$. The other measured data showed $0.04 \mu \mathrm{Gy} / \mathrm{h}$ in Fukushima city, 0.04-0.06 $\mu \mathrm{Gy} / \mathrm{h}$ in Koriyama city, $0.04-0.05 \mu \mathrm{Gy} / \mathrm{h}$ in Shirakawa city, $0.04-0.05 \mu \mathrm{Gy} / \mathrm{h}$ in Aizuwakamatsu city, $0.02-0.04 \mu \mathrm{Gy} / \mathrm{h}$ in Minami-aizu town, $0.05 \mu \mathrm{Gy} / \mathrm{h}$ in Minami-soma town and $0.05-0.06 \mu \mathrm{Gy} / \mathrm{h}$ in Iwaki city $^{5}$. As the background dose rate, $0.04 \mu \mathrm{Gy} / \mathrm{h}$ as the median value among them was used for the background subtractions. Actually, the background effective dose rate was set to $0.03 \mu \mathrm{Sv} / \mathrm{h}$ by applying the conversion coefficient from air kerma to effective dose.
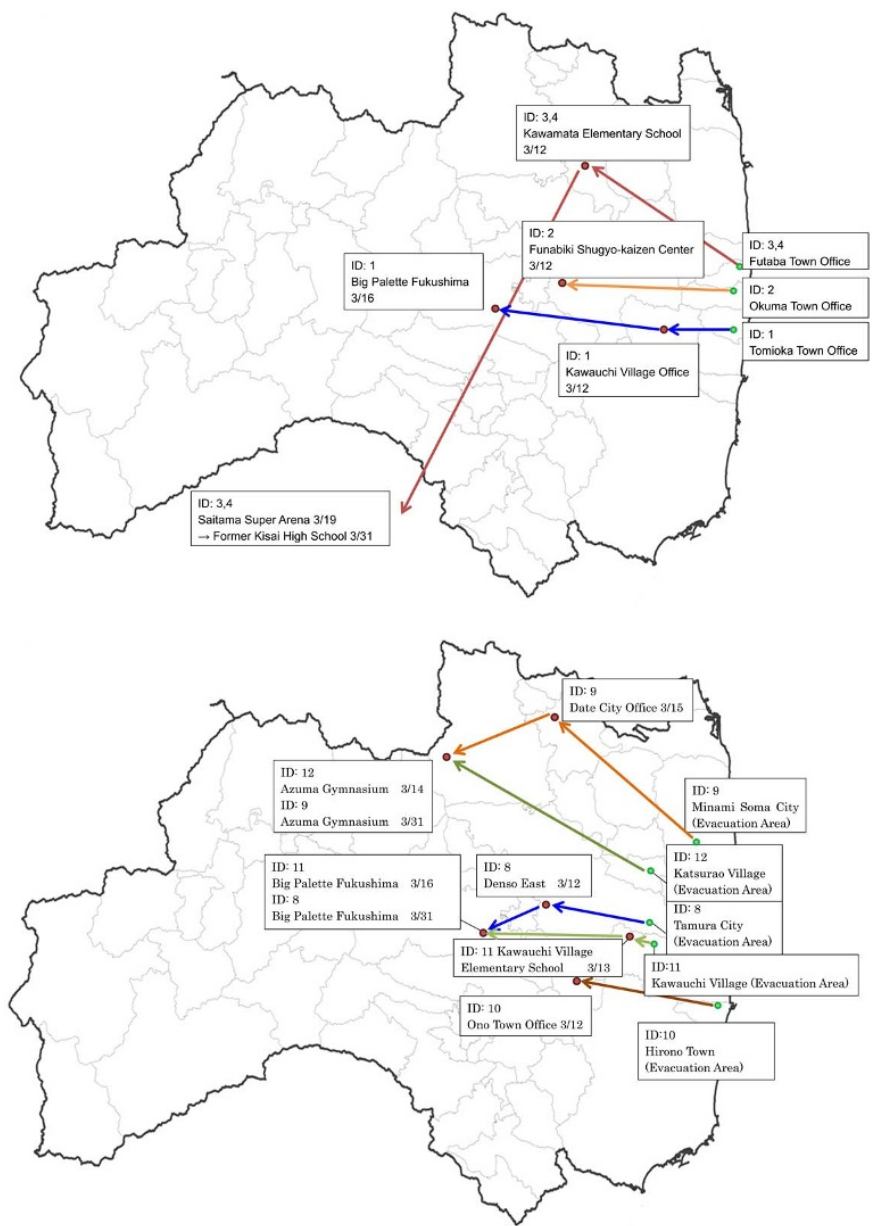

The monitoring data are measured in terms of ambient dose equivalent $H^{*}(10)$, which is an operational guantity. On the other hand effective dose $\mathrm{E}$ is protection quantity and used for risk assessment of human health ${ }^{13,14}$. Then the conversion factors from ambient dose equivalent to effective dose $\frac{\dot{E}}{H^{*}(10)}$ were used for

calculations in the system. According to the previous study ${ }^{15}$, the main radionuclides emitted from the Fukushima Dai-ichi NPP were Xe-133, Te-132 and I-132 at the early stage of the accident, and Cs-134 and Cs-137 afterwards. Adding I-131 and Te-129m to these radionuclides, the conversion coefficients of effective dose per ambient dose equivalent for each radionuclide were calculated in the following steps;

1) Calculate conversion factors of $\left(\frac{E}{\phi}\right)$ for isotropic irradiation (ISO) and $\left(\frac{H^{*}(10)}{\phi}\right)$ for the energy of the i-th gamma ray emitted from a radionuclide, $\left(\frac{E}{\phi}\right)_{i}$ and $\left(\frac{H^{*}(10)}{\phi}\right)_{i}$, according to the ICRP publication 74 with the log-log interpolation method. Here, $\phi$ is the fluence of gamma ray.

2) Calculate conversion factors of $\left(\frac{E}{\phi}\right)$ for isotropic irradiation (ISO) and $\left(\frac{H^{*}(10)}{\phi}\right)$ for each nuclide, $\left(\frac{E}{\phi}\right)_{\text {nuclide }}$ and $\left(\frac{H^{*}(10)}{\phi}\right)_{\text {nuclide }}$, by the following equations:

$$
\begin{gathered}
\left(\frac{E}{\phi}\right)_{\text {nuclide }}=\frac{\sum_{i}\left(\left(\frac{E}{\phi}\right)_{i} \times R_{i}\right)}{\sum_{i} R_{i}} \\
\left(\frac{H^{*}(10)}{\phi}\right)_{\text {nuclide }}=\frac{\sum_{i}\left(\left(\frac{H^{*}(10)}{\phi}\right)_{i} \times R_{i}\right)}{\sum_{i} R_{i}},
\end{gathered}
$$

where $R_{i}$ is the emission ratio of the $\mathrm{i}$-th gamma ray emitted from a radionuclide.

3) Divide $\left(\frac{E}{\phi}\right)_{\text {nuclide }}$ by $\left(\frac{H^{*}(10)}{\phi}\right)_{\text {nuclide }}$
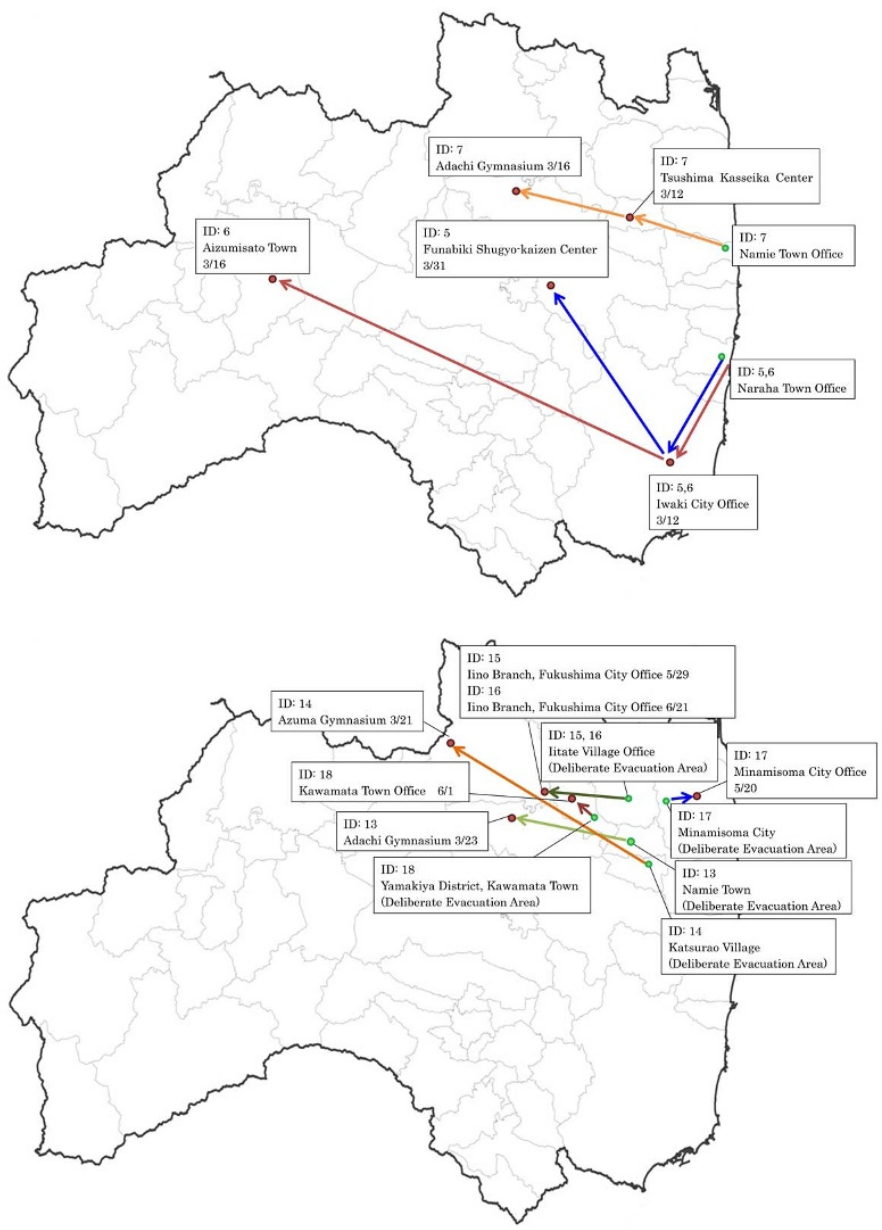

Figure $5 \mid$ Assumed 18 evacuation patterns of the residents. Basic maps were created by using ArcGIS of ESRI. 
The calculated $\frac{E}{H^{*}(10)}$ for each nuclide is shown in Table 2 . In our estimation, the value of 0.6 was adopted as $\frac{E}{H^{*}(10)}$ for adult, rounding the value of 0.59 which is the maximum among the radionuclides. Actually, since there are differences of tissue weighting factors of effective dose between ICRP Publication $60^{13}$ and $103^{14}$, estimated effective doses is not the same exactly depending on the publication. However, according to the reference ${ }^{16}$, these values are similar for isotropic irradiation. Hence the correction between them was not considered in the system.

Finally, the effective dose (for adult) rate maps with $d(h, m)$, can be obtained in a time series from 15 March to 11 July, 2011 in all parts of Fukushima prefecture and a part of neighboring four prefectures (Miyagi, Yamagata, Tochigi, and Ibaraki) painted in pink and blue in Fig. 3 .

Calculation of effective dose. The external effective doses were calculated for three different situations: staying indoors/outdoors and moving from one place to another.

When one stays indoors, the dose reduction should be considered because buildings have shielding effects against radiation exposures depending on their materials and thickness of the walls. In the TECDOC $225^{15}$ by the International Atomic Energy Agency (IAEA), representative reduction factors for cloud, $r_{c}$, and ground sources, $r_{g}$, are shown. From 12 to $14 \mathrm{March}$, the radio nuclides in the plume have contributed to the dose rates in the environment. On the contrary, the radionuclides on the ground were major sources of the exposures because of the rain or snow falls on 15 March in Fukushima. In the external dose calculation system, the reduction factors for cloud source in TECDOC 225 were adopted for the calculation from 12 to 14 March. The values used in this system were $0.9,0.6$, and 0.2 for one or two story wood frame house, one or two story concrete house or building, and three or more story concrete building, respectively. For the period of $15 \mathrm{March}$ and after, the factors for deposited radioactivity were applied in the calculations. The values were $0.4,0.2$ and 0.1 for one or two story wood frame house, one or two story concrete house or building, and three or more story concrete building, respectively.

Dose rates during a move may change depending on the location. The dose exposed during the move is simply calculated as the product of mean effective dose rate at the start and end points multiplying the hours of the move.

Finally, when the mesh number including the place, $j$ is $m(j)$, and dose reduction factor correspond to the type of the building, $k_{j}$ is $r_{c / g}\left(k_{j}\right)$, dose for stay on the date, $h$, $E_{\text {stay }, h}$ and dose for the move on the date, $h, E_{\text {move } h}$ express as the following equations, respectively.

$$
\begin{gathered}
E_{s t y, h}=\sum_{j}\left\{d(h, m(j)) \times\left[t_{\text {in, } j} \times r_{c / g}\left(k_{i}\right)+t_{\text {out }, j}\right]\right\} \\
E_{\text {move } h}=\sum_{j}\left\{\frac{d(h, m(j))+d(h, m(j+1))}{2} \times t_{\text {move }, j \rightarrow j+1}\right\}
\end{gathered}
$$

The effective dose from March 12 to July 11, 2011, E, can be obtained with the following equation.

$$
E=\sum_{h}\left(E_{\text {stay }, h}+E_{\text {move }, h}\right)
$$

The conversion coefficients from ambient dose equivalent to effective dose E/ $H^{*}(10)$ are higher as the age is lower, because the shielding effect increases with the volumes of body stem ${ }^{18}$. When the maximum conversion coefficient was used for a conservative estimation, conversion coefficient, $C_{\text {age }}$ (age coefficient) was expressed as the following linear function of age, $y$, between 1 and 15 years old (Fig. 4).

$$
C_{\text {age }}(y)=-0.0144 \times y+1.27
$$

The effective dose for children can be calculated by multiplying $E$ by $C_{\text {age. }}$.

Eighteen patterns of the evacuations. For roughly grasping and estimating the doses, 18 evacuation patterns of the residents from the area within $20 \mathrm{~km}$ radius of the NPP, and the deliberate evacuation area $^{2}$ were assumed by considering the actual evacuation information before using the survey data (Fig. 5).

Since the traffic conditions were worse compared to those in ordinary situation, the moving speeds of evacuations were assumed to be $10 \mathrm{~km} / \mathrm{h}$ for the moving inside the prefecture, and $30 \mathrm{~km} / \mathrm{h}$ for those from Fukushima to other prefectures. Until the end of March, the ordinary traffic conditions were considered. For the time of activities outside the houses or buildings, it is assumed that the residents stayed for 4 hours a day before the evacuations, and 2 hours a day after the evacuations.

The two places where the residents started to evacuate were assumed; the representative place such as town office, and/or the places where the doses were highest in the town until when they evacuated.

1. Nuclear Emergency Response Headquarters of Government of Japan (2011a). Report of the Japanese Government to the IAEA Ministerial Conference on Nuclear Safety-The Accident at TEPCO's Fukushima Nuclear Power Stations. June (2011).
2. Nuclear Emergency Response Headquarters of Government of Japan (2011b). Additional Report of the Japanese Government to the IAEA-The Accident at TEPCO's Fukushima Nuclear Power Stations-(Second Report). September (2011).

3. Yasumura, S., Hosoya, M., Yamashita, S., Kamiya, K. \& Abe, M. et al. Study protocol for the Fukushima health management survey. J Epidemiol. 22 (5), 375-383 (2012).

4. Ministry of Education, Culture, Sports, Science and Technology. SPEEDI System for Prediction of Environmental Emergency Dose Information. Pamphlet of SPEEDI (2007).

5. Monitoring data in Fukushima prefecture. http://www.pref.fukushima.jp/j/ 7houbu0311-0331.pdf (in Japanese: Accessed on 20 February 2013).

6. Monitoring data in Fukushima prefecture. http://www.radioactivity.nsr.jo.jp/ja/ contents/5000/4106/24/1304764_0406_01.pdf (in Japanese: Accessed on 20 February 2013).

7. Monitoring data in Fukushima prefecture. http://www.pref.fukushima..jp/j/ 20-50km0315-0331.pdf (in Japanese: Accessed on 20 February 2013).

8. Saito, K., Petoussi-Henss, N. \& Zankl, M. Calculation of the effective dose and its variation from environmental gamma ray sources. Health Physics. 74 (6), 698-706 (1998).

9. International Commission on Radiological Protection. Conversion Coefficients for use in Radiological Protection against External Radiation. ICRP Publication 74. Ann. ICRP 26 (3-4), (1996).

10. MELCOR: a computer code for analyzing severe accidents in nuclear power plants and the design basis accidents for advanced power plant applications. http:// melcor.sandia.gov/ (accessed on 20 February 2013).

11. Nuclear Regulation Authority. Calculation results of SPEEDI. http:// www.nsr.go.jp/archive/nisa/earthquake/speedi/230724/230724.html (in Japanese: Accessed on 20 February 2013).

12. Monitoring data in Fukushima prefecture. http://www.pref.fukushima.jp/ nuclear/old/kanshi/H22-3.pdf (in Japanese: Accessed on 20 February 2013).

13. International Commission on Radiological Protection. 1990 Recommendations of the International Commission on Radiological Protection. ICRP Publication 60. Ann. ICRP 21 (1-3), (1991).

14. International Commission on Radiological Protection. The 2007 Recommendations of the International Commission on Radiological Protection. ICRP Publication 103. Ann. ICRP 37 (2-4), (2007).

15. Matsumura, H., Saito, K., Ishioka, J. \& Uwamino, Y. Diffusion of Radioactive Materials from Fukushima Daiichi Nuclear Power Station Obtained by GammaRay Measurements on Expressways. Transactions of the Atomic Energy Society of Japan 10 (3), 152-162 (2011). (in Japanese).

16. Schlattl, H., Zankl, M. \& Petoussi-Henss, N. Organ dose conversion coefficients for voxel models of the reference male and female from idealized phantom exposures. Phys. Med. Biol. 52, 2123-2145 (2007).

17. International Atomic Energy Agency, Planning for off-site response to radiation accidents in nuclear facilities, IAEA-TECDOC225 (1979).

18. Yamaguchi, Y. Age-dependent effective doses for external photons. Radiat. Prot. Dosim. 55, 123-129 (1994).

\section{Acknowledgements}

We would like to thank members of Dose Estimation Committee of Fukushima prefecture, Prof. Takashi Nakamura of Tohoku University, Dr. Yoshiro Uwamino of RIKEN, Dr. Sakae Kinase of Japan Atomic Energy Agency, Prof. Takayuki Takahashi of Fukushima University, Prof. Naoki Matsuda of Nagasaki University and Prof. Hiromi Yamazawa of Nagoya University, for their helpful suggestions and discussions on the external dose estimation.

\section{Author contributions}

M.A. conducted this project. All authors performed the conceptual design of this system, and K.A. and S.Y. have developed the current system in the Fukushima health management survey. K.A. and S.Y. wrote the text and made tables and figures. All authors reviewed the manuscript.

\section{Additional information}

Competing financial interests: The authors declare no competing financial interests.

License: This work is licensed under a Creative Commons

Attribution-NonCommercial-NoDerivs 3.0 Unported License. To view a copy of this license, visit http://creativecommons.org/licenses/by-nc-nd/3.0/

How to cite this article: Akahane, K. et al. NIRS external dose estimation system for Fukushima residents after the Fukushima Dai-ichi NPP accident. Sci. Rep. 3, 1670; DOI:10.1038/srep01670 (2013). 